\title{
Magnus-type integrator for semilinear delay equations with an application to epidemic models
}

\author{
Petra Csomós \\ Institute of Mathematics, Eötvös Loránd University, Pázmány Péter st. 1/C, H-1117 Budapest, Hungary
}

\section{A R T I C L E I N F O}

\section{Article history:}

Received 9 January 2019

Received in revised form 3 April 2019

\section{Keywords:}

Magnus method

Convergence analysis

Quasilinear delay equation

Delayed epidemic model

SIR model

\begin{abstract}
A B S T R A C T
We present a numerical method based on the Magnus series expansion, and show its second-order convergence when applied to a system of quasilinear delay equations. As an application, we take the delayed epidemic model and illustrate our results with numerical experiments.
\end{abstract}

(C) 2019 Elsevier B.V. All rights reserved.

\section{Introduction}

Epidemics affect everyone's lives. Due to frequent and long-distance traveling infectious diseases can spread rapidly, demanding more and more victims. Moreover, altered conditions caused by the climate change result in temporal and spatial changes in the source of infections. It is therefore of high importance to predict the outbreaks as accurately as possible. Besides collecting the data on past and present epidemics, the use of mathematical models offers a forecast capturing the main characteristics of an epidemics (such as the number of infected individuals). Since mathematical epidemic models are of a form of rather complex ordinary or partial differential equations, their exact solution cannot be determined. Instead, an approximation is computed by applying certain numerical methods.

The mathematical modeling of epidemics originates from the early twentieth century. A summary of the first attempts can be found in [1, Section 1.4]. Already Sir Ronald Ross, being awarded the Nobel Prize for the discovery of the malarial parasite, was convinced about the need of mathematical tools in epidemiology (see [1] and the references therein). The first epidemics models were proposed by Kermack and McKendrick in [2]. Their seminal work has lead to a large amount of completion and development of their model, making epidemic modeling a research field being promising in terms of social benefits.

An important direction of developing epidemic models is the consideration of latent period, the time when a person is infected but is not infective, that is, the time from when the infected is really able to infect another individual. Incorporating the latent period leads to a system of differential equations with delay. In case of delayed epidemic models, the temporal change in the model's unknown quantities (usually the number of susceptible, infected, and recovered individuals) do not only depend on their values at the actual time level but also on their values in the past (i.e. the latent period ago). Solution of delayed epidemic models needs efficient numerical methods which provides fast and accurate results.

In the present paper we propose a numerical method based on the use of Magnus method being originally developed for nonautonomous problems by Magnus in [3]. We show that the delayed epidemic models can be written in a quasilinear

E-mail address: csomos@cs.elte.hu. 
form, and our approach leads to a positivity preserving and convergent numerical method which computes the numerical solution in an efficient way.

In Section 2 we introduce the Magnus-type integrator being studied in the present paper. Section 3 deals with the positivity preservation and convergence of Magnus-type integrator when applied to quasilinear delay equations. In Section 4 we show that our results concerning the convergence and positivity preservation are valid for delayed epidemic models. In Section 5 numerical experiments are presented to illustrate the theoretical results.

\section{Magnus-type integrator}

In this section we introduce the Magnus-type integrator, and present three important convergence results from the literature which will be needed later on. For an arbitrary $d \in \mathbb{N}$, we consider the following nonautonomous evolution equation for the continuously differentiable unknown function $Y: \mathbb{R} \rightarrow \mathbb{R}^{d}$ where $A(t): \mathbb{R}^{d} \rightarrow \mathbb{R}^{d}$ is a linear operator for all $t \geq 0$ and $w \in \mathbb{R}^{d}$ :

$$
\left\{\begin{array}{l}
Y^{\prime}(t)=A(t) Y(t), \quad t \geq 0 \\
Y(0)=Y_{0}
\end{array}\right.
$$

If problem (1) is well-posed, then for all $t \geq 0$ there exists a linear operator $\Omega(t, 0): \mathbb{R}^{d} \rightarrow \mathbb{R}^{d}$, such that the exact solution has the form $Y(t)=\mathrm{e}^{\Omega(t, 0)} Y_{0}$ for all $t \geq 0$. We note that $\left(\mathrm{e}^{\Omega\left(T_{2}, T_{1}\right)}\right)_{T_{1} \leq T_{2}}$ is an evolution family possessing the following properties (see e.g. in $[4,5]$ ):

(a) $\Omega(T, T)$ is the identity for all $T \geq 0$,

(b) $\mathrm{e}^{\Omega\left(T_{3}, T_{2}\right)} \mathrm{e}^{\Omega\left(T_{2}, T_{1}\right)}=\mathrm{e}^{\Omega\left(T_{3}, T_{1}\right)}$ holds for all $0 \leq T_{1} \leq T_{2} \leq T_{3}$,

(c) the mapping $\left(T_{1}, T_{2}\right) \mapsto \mathrm{e}^{\Omega\left(T_{2}, T_{1}\right)}$ is strongly continuous,

(d) $\left\|\mathrm{e}^{\Omega\left(T_{2}, T_{1}\right)}\right\| \leq M \mathrm{e}^{\Omega\left(T_{2}-T_{1}\right)}$ holds for some $M \geq 1, \Omega \in \mathbb{R}$ and for all $0 \leq T_{1} \leq T_{2}$.

An approximation of operator $\Omega(t, 0)$ is based on the infinite series expansion of $Y(t)$ introduced in his seminal paper [3] by Magnus, and it reads for all $m \in \mathbb{N}$ as:

$$
\begin{aligned}
\Omega^{[0]}(t, 0) & =0 \\
\Omega^{[m]}(t, 0) & =\int_{0}^{t} \sum_{k=0}^{\infty} \frac{B_{k}}{k !} \operatorname{ad}_{\Omega^{[m-1]}(s, 0)}^{k} A(s) \mathrm{d} s,
\end{aligned}
$$

where $B_{k}, k \in \mathbb{N}_{0}$ are the Bernoulli numbers and $\operatorname{ad}_{\Omega}^{[m]} A:=\left[\Omega, \operatorname{ad}_{\Omega}^{[m-1]} A\right]$ is the iterated commutator with ad ${ }_{\Omega}^{[0]}$ being the identity. The convergence of the Magnus expansion (2) has been extensively investigated in the literature, see e.g. in [6,7], and the review [8]. We cite now the result of Moan and Niesen which gives a sufficient condition for the convergence.

Theorem 1 (Theorem 3 in [9]). Let $A(t)$ be a real integrable matrix, and let $Y$ denote the solution of problem (1). If the relation

$$
\int_{0}^{t}\|A(s)\|_{2} \mathrm{~d} s<\pi
$$

holds, then the Magnus series (2) converges, and its sum $\Omega(t, 0)$ satisfies $\mathrm{e}^{\Omega(t, 0)} Y_{0}=Y(t)$.

Based on series (2), the corresponding approximate solution has the form

$$
Y^{[m]}(t)=\mathrm{e}^{\Omega^{[m]}(t, 0)} Y_{0}
$$

and is called the Magnus method. We cite the corresponding convergence result.

Theorem 2 (Theorem. 2.1 in [10]). For the Magnus method (3), there exists a constant $C>0$, being independent of $t$, such that $\left\|Y(t)-Y^{[m]}(t)\right\| \leq C t^{m+1}$ holds for all $t \geq 0$.

In what follows we consider the case $m=1$, and use the notation $Y^{[1]}(t)=: y(t)$. Then the Magnus method (3) with $y_{0}=Y_{0}$ is written as

$$
y(t)=\mathrm{e}^{\Omega^{[1]}(t, 0)} y_{0} \quad \text { with } \quad \Omega^{[1]}(t, 0)=\int_{0}^{t} A(s) \mathrm{d} s .
$$

For further use, we give the general formula for all $T, \Delta T \geq 0$ real numbers:

$$
\Omega^{[1]}(T+\Delta T, T)=\int_{0}^{\Delta T} A(T+s) \mathrm{d} s .
$$

We note that the additivity of the integral implies the following relation:

$$
\Omega^{[1]}(T+\Delta T, 0)=\Omega^{[1]}(T, 0)+\Omega^{[1]}(T+\Delta T, T) .
$$


In order to define a numerical method later, we need another form of Magnus method (4). We define a time step $\tau>0$ and the time levels $t_{n}=n \tau$ for all $n \in \mathbb{N}_{0}$. Then formulae (4), (5), and (6) with $T=t_{n}$ and $\Delta T=\tau$ lead to the following form of the Magnus method:

$$
y\left(t_{n+1}\right)=\mathrm{e}^{\Omega^{[1]}\left(t_{n}+\tau, 0\right)} y_{0}=\mathrm{e}^{\Omega^{[1]}\left(t_{n}+\tau, t_{n}\right)} \mathrm{e}^{\Omega^{[1]}\left(t_{n}, 0\right)} y_{0}=\mathrm{e}^{\Omega^{[1]}\left(t_{n}+\tau, t_{n}\right)} y\left(t_{n}\right)=\exp \left(\int_{0}^{\tau} A\left(t_{n}+s\right) \mathrm{d} s\right) y\left(t_{n}\right) .
$$

The Magnus method (7) still consists of an integral which we need to approximate by a quadrature rule to get a numerical method. We use the midpoint rule and get

$$
y\left(t_{n+1}\right) \approx \mathrm{e}^{\tau A\left(t_{n}+\frac{\tau}{2}\right)} y\left(t_{n}\right) .
$$

We denote the approximation of $y\left(t_{n}\right)$ at the time level $t_{n}=n \tau$ by $\widehat{y}_{n}$ for all $n \in \mathbb{N}_{0}$. Then we obtain the Magnus-type integrator as follows:

$$
\widehat{y}_{n+1}=\mathrm{e}^{\tau A\left(t_{n}+\frac{\tau}{2}\right)} \widehat{y}_{n}
$$

with $\widehat{y}_{0}=Y_{0}$. For further reference we cite here the results of González et al. reformulated for operators acting on the space $\mathbb{R}^{d}$.

Theorem 3 (Theorem 2. in [11]). Suppose that the operator $A(t): \mathbb{R}^{d} \rightarrow \mathbb{R}^{d}$ is uniformly sectorial for $t \in[0, T]$. Thus, there exist constants $a \in \mathbb{R}, 0<\Gamma<\pi / 2$, and $M_{1} \geq 1$ such that $A(t)$ satisfies the resolvent condition

$$
\left\|(A(t)-\lambda I)^{-1}\right\| \leq \frac{M_{1}}{|\lambda-a|}
$$

for any $\lambda$ lying in the complement of the sector $S_{\Gamma}(a)=\{\lambda \in \mathbb{C}:|\arg (a-\lambda)| \leq \Gamma\} \cup\{a\}$. Suppose further that $A$ is Lipschitz continuous, that is, there is a constant $M_{2}>0$ such that the estimate

$$
\|A(t)-A(s)\| \leq M_{2}(t-s)
$$

holds for all $0 \leq s \leq t \leq T$. Then there exists a constant $C>0$, independent of $n$ and $\tau$, such that the error estimate

$$
\left\|Y\left(t_{n}\right)-\widehat{y}_{n}\right\| \leq C \tau^{2}\left(\left\|g^{\prime}\right\|_{\infty}+\left\|g^{\prime \prime}\right\|_{\infty}\right)
$$

holds with

$$
\begin{aligned}
g_{n}(t) & =\left(A(t)-A\left(t_{n}+\frac{\tau}{2}\right)\right) Y(t) \\
\left\|g^{\prime}\right\|_{\infty} & =\max _{n \in \mathbb{N}_{0}} \max _{t \in\left[t_{n}, t_{n+1}\right]}\left\|g_{n}^{\prime}(t)\right\| \\
\left\|g^{\prime \prime}\right\|_{\infty} & =\max _{n \in \mathbb{N}_{0}} \max _{t \in\left[t_{n}, t_{n+1}\right]}\left\|g_{n}^{\prime \prime}(t)\right\|
\end{aligned}
$$

and $t_{n+1} \leq T$, whenever the right hand-side exists.

From now on $C>0$ denotes a generic constant.

\section{Magnus-type integrator for quasilinear delay equations}

We present now how the Magnus-type integrator (8) can be applied to quasilinear delay equations, show that it preserves the positivity, and prove its second-order convergence.

Delay problems arise in numerous application fields where the system's temporal change depends on the system's past state(s) as well. They represent differential equations where the derivative does not only depend on the actual value of the unknown function but also on its values in the past. In what follows we treat quasilinear equations where operator $Q(w): \mathbb{R}^{d} \rightarrow \mathbb{R}^{d}$ is linear for all $w \in \mathbb{R}^{d}, \delta>0$ is the time delay parameter, and $\phi:[-\delta, 0] \rightarrow \mathbb{R}^{d}$ is a given continuous function representing the history of the system. Then we are for the continuous unknown function $Y: \mathbb{R} \rightarrow \mathbb{R}^{d}$ satisfying the quasilinear delay equation of the form

$$
\left\{\begin{aligned}
Y^{\prime}(t) & =Q(Y(t-\delta)) Y(t), & t>0, \\
Y(s) & =\phi(s), & s \in[-\delta, 0] .
\end{aligned}\right.
$$

We note that for $\delta>0$, the value $Y(t-\delta)$ is given for all $t \geq 0$. Therefore, the linear operator $Q(Y(t-\delta))$ is also known. The quasilinear delay equation (9) fits in the framework of problem (1) with the operator defined as

$$
A(t)=Q(Y(t-\delta))
$$

for all $t \geq 0$. For the operator $A$ defined in (10), an arbitrary $\tau>0$, and $t_{n}=n \tau, n \in \mathbb{N}_{0}$, the Magnus method (7) has the form

$$
y\left(t_{n+1}\right)=\exp \left(\int_{0}^{\tau} Q(Y(s-\delta)) d s\right) y\left(t_{n}\right),
$$


where $Y$ is given on the interval $[-\delta, 0]$. The exact solution $Y$ is again approximated by the Magnus method (7) itself. Altogether we obtain

$$
\begin{aligned}
y\left(t_{n+1}\right)= & \exp \left(\int_{0}^{\tau} Q\left(\widetilde{y}_{n}(s)\right) \mathrm{d} s\right) y\left(t_{n}\right) \\
\tilde{y}_{n}(s) & = \begin{cases}\phi\left(t_{n}-\delta+s\right) & \text { with } \\
\exp \left(\int_{0}^{s} Q\left(\phi\left(t_{n}-2 \delta+\xi\right)\right) \mathrm{d} \xi\right) y\left(t_{n}-\delta\right) & \text { for } t_{n}+s \in[\delta, 2 \delta), \\
\exp \left(\int_{0}^{s} Q\left(y\left(t_{n}-2 \delta+\xi\right)\right) \mathrm{d} \xi\right) y\left(t_{n}-\delta\right) & \text { for } t_{n}+s \geq 2 \delta .\end{cases}
\end{aligned}
$$

The Magnus-type integrator is derived by approximating the integrals by appropriate quadrature rules. In the formula for $y\left(t_{n+1}\right)$ we use the midpoint rule:

$$
y\left(t_{n+1}\right) \approx \mathrm{e}^{\tau Q\left(\widetilde{y}_{n}\left(\frac{\tau}{2}\right)\right)} y\left(t_{n}\right),
$$

and in the approximation of $\widetilde{y}_{n}\left(\frac{\tau}{2}\right)$ we use the left rectangle rule:

$$
\tilde{y}_{n}\left(\frac{\tau}{2}\right) \approx \begin{cases}\phi\left(t_{n}-\delta+\frac{\tau}{2}\right) & \text { for } t_{n} \in[0, \delta), \\ \mathrm{e}^{\frac{\tau}{2} Q\left(\phi\left(t_{n}-2 \delta\right)\right)} y\left(t_{n}-\delta\right) & \text { for } t_{n} \in[\delta, 2 \delta), \\ \mathrm{e}^{\frac{\tau}{2} Q\left(y\left(t_{n}-2 \delta\right)\right)} y\left(t_{n}-\delta\right) & \text { for } t_{n} \geq 2 \delta .\end{cases}
$$

In order to proceed, we choose an arbitrary number $N \in \mathbb{N}$, and define the time step as $\tau=\delta / N$. We suppose that the Magnus series converges, e.g. $A(t)$ is integrable and $\tau$ satisfies the convergence criterion in Theorem 1:

$$
\int_{0}^{\tau}\|A(s)\|_{2} \mathrm{~d} s<\pi
$$

Then the Magnus-type integrator for quasilinear delay equation (9) has the form

$$
\begin{aligned}
y_{n+1} & =\mathrm{e}^{\tau Q\left(\widetilde{y}_{n}\right)} y_{n} \quad \text { with } \\
\tilde{y}_{n} & = \begin{cases}\phi\left(t_{n}-\delta+\frac{\tau}{2}\right) & \text { for } n=0,1, \ldots, N-1, \\
\mathrm{e}^{\frac{\tau}{2} Q\left(\phi\left(t_{n}-2 \delta\right)\right)} y_{n-N} & \text { for } n=N, \ldots, 2 N-1, \\
\mathrm{e}^{\frac{\tau}{2} Q\left(y_{n-2 N}\right) y_{n-N}} & \text { for } n \geq 2 N .\end{cases}
\end{aligned}
$$

In what follows we analyze the Magnus-type integrator (13) in terms of positivity preservation and convergence, moreover, in Section 4 we apply it to epidemic models.

In many physical/chemical/biological applications the unknown function should be positive (e.g. mass, pressure, concentration, population), unless one gets unreliable solutions. Thus, it is desirable that the numerical method preserves the sign of the solution, too. In what follows we give a sufficient condition for the positivity preservation of Magnus-type integrator (13).

\section{Definition 4.}

(a) A vector having nonnegative elements only, is called a positive vector.

(b) A matrix $W \in \mathbb{R}^{d \times d}$ is called a Metzler matrix if its off-diagonal elements are nonnegative.

Remark 5. For $d \in \mathbb{N}$, let $W \in \mathbb{R}^{d \times d}$ be an arbitrary matrix and $w \in \mathbb{R}^{d}$ be a positive vector. Then [12, Lemma 5.3.a] states that $\mathrm{e}^{W} w$ is a positive vector if and only if $\mathrm{e}^{W}$ has only nonnegative elements. Moreover, [12, Thm. 7.1] states that $\mathrm{e}^{W}$ has only nonnegative elements if and only if $W$ is a Metzler matrix.

Corollary 6. For $d \in \mathbb{N}$, let $W \in \mathbb{R}^{d \times d}$ be an arbitrary matrix and $w \in \mathbb{R}^{d}$ be a positive vector. Remark 5 implies that $\mathrm{e}^{W} w$ is a positive vector if and only if $W$ is a Metzler matrix.

Proposition 7. Let $Q: \mathbb{R}^{d} \rightarrow \mathbb{R}^{d \times d}$ be a function such that $Q(w)$ is a Metzler matrix for all positive vectors $w \in \mathbb{R}^{d}$. Then the Magnus-type integrator (13) preserves the positivity, that is, $y_{n+1}$ is a positive vector for positive vectors $y_{n}$ and $\phi(t)$, $t \in[-\delta, 0]$.

Proof. We remark first that if $Q(w)$ is a Metzler matrix then $t Q(w)$ is that as well for all $t \geq 0$. The Magnus-type integrator (13) has the form

$$
y_{n+1}=\mathrm{e}^{\tau Q\left(\widetilde{y}_{n}\right)} y_{n} .
$$


By Corollary 6 it suffices to show that the vector

$$
\widetilde{y}_{n}= \begin{cases}\phi\left(t_{n}-\delta+\frac{\tau}{2}\right) & \text { for } n=0,1, \ldots, N-1, \\ \mathrm{e}^{\frac{\tau}{2} Q\left(\phi\left(t_{n}-2 \delta\right)\right)} y_{n-N} & \text { for } n=N, \ldots, 2 N-1, \\ \mathrm{e}^{\frac{\tau}{2} Q\left(y_{n-2 N}\right) y_{n-N}} & \text { for } n \geq 2 N\end{cases}
$$

is positive for all $n \in \mathbb{N}_{0}$. We distinguish the following cases.

(i) For $n=0, \ldots, N-1$, the vector $\widetilde{y}_{n}=\phi\left(t_{n}-\delta+\frac{\tau}{2}\right)$ is positive.

(ii) For $n=N$, we have $\widetilde{y}_{N}=\mathrm{e}^{\frac{\tau}{2} Q(\phi(-\delta))} y_{0}$, where $\phi(-\delta)$ and $y_{0}$ are positive vectors by assumption. Thus, $\frac{\tau}{2} Q(\phi(-\delta))$ is a Metzler matrix and therefore $\tilde{y}_{N}$ is a positive vector by Corollary 6 .

(iii) Let $n=N+1, \ldots, 2 N-1$. Then $\phi\left(t_{n}-2 \delta\right)$ is a positive vector and hence $\frac{\tau}{2} Q\left(\phi\left(t_{n}-2 \delta\right)\right)$ is a Metzler matrix. Moreover, it holds that for indices

$$
m:=n-N-1=0, \ldots, N-2
$$

the vector $y_{m+1}=\mathrm{e}^{\tau Q\left(\widetilde{y}_{m}\right)} y_{n-N}=\mathrm{e}^{\tau Q\left(\phi\left(t_{m}-\delta+\frac{\tau}{2}\right)\right)} \phi\left(t_{m}-\delta\right)$ is positive by step (i) and Corollary 6. Hence, $y_{n-N}$ is a positive vector, too.

(iv) For indices $n \geq 2 N$ we proceed by induction. We saw that there was an index $k \in \mathbb{N}_{0}$ such that vector $\tilde{y}_{n}$ was positive for all $n=0, \ldots, k$. Therefore the assumption on $Q$ and Corollary 6 imply that

$$
\text { vector } y_{n+1} \text { is positive for all } n=0, \ldots, k \text {. }
$$

Our aim is to show that $\widetilde{y}_{k+1}$ is a positive vector. The cases $k<2 N$ were shown in steps (i)-(iii), therefore, we consider the case $k \geq 2 N$. Then formula (14) yields

$$
\tilde{y}_{k+1}=\mathrm{e}^{\frac{\tau}{2} Q\left(y_{k-2 N}\right)} y_{k-N} .
$$

Since $n_{1}:=k-2 N-1<k$ and $n_{2}:=k-N-1<k$, the assertion (15) assures that the vectors $y_{n_{1}+1}=y_{n-2 N}$ and $y_{n_{2}+1}=y_{n-N}$ are positive. Therefore, $\widetilde{y}_{k+1}$ is positive again by Corollary 6 . Then the positivity of $\tilde{y}_{n}$ for all $n \in \mathbb{N}_{0}$ follows by induction.

Since we obtained that $\widetilde{y}_{n}$ is a positive vector for all $n \in \mathbb{N}$, Corollary 6 implies the positivity of vector $y_{n+1}=\mathrm{e}^{\tau Q\left(\widetilde{y}_{n}\right)} y_{n}$ for all $n \in \mathbb{N}$, as well, which was to prove.

Our next aim is to analyze under which conditions the Magnus-type integrator (13) is convergent to the exact solution of problem (9). In what follows, for a function $F: \mathbb{R} \rightarrow \mathbb{R}^{d}$ and a number $p>0$, the notation $F(\tau)=\mathcal{O}\left(\tau^{p}\right)$ means that there exists a constant $C>0$ such that the relation $\|F(\tau)\| \leq C \tau^{p}$ holds for all $\tau \in[0, T]$. We need a technical lemma.

Lemma 8. For matrices $W, Z \in \mathbb{R}^{d \times d}$ and $K:=\max \{\|W\|,\|Z\|\}$, the following estimate holds:

$$
\left\|\mathrm{e}^{W}-\mathrm{e}^{Z}\right\| \leq \mathrm{e}^{K}\|W-Z\| .
$$

Proof. By the matrix exponential and the telescopic identity we can write

$$
\begin{aligned}
\left\|\mathrm{e}^{W}-\mathrm{e}^{Z}\right\| & =\left\|\sum_{k=0}^{\infty} \frac{W^{k}}{k !}-\sum_{k=0}^{\infty} \frac{Z^{k}}{k !}\right\|=\left\|\sum_{k=0}^{\infty} \frac{1}{k !}\left(W^{k}-Z^{k}\right)\right\|=\left\|\sum_{k=0}^{\infty} \frac{1}{k !} \sum_{\ell=0}^{k-1} W^{k-1-\ell}(W-Z) Z^{\ell}\right\| \\
& \leq \sum_{k=0}^{\infty} \frac{1}{k !} \sum_{\ell=0}^{k-1}\|W\|^{k-1-\ell}\|W-Z\|\|Z\|^{\ell} \leq \sum_{k=0}^{\infty} \frac{1}{k !} \sum_{\ell=0}^{k-1} K^{k-1}\|W-Z\|=\sum_{k=0}^{\infty} \frac{k K^{k-1}}{k !}\|W-Z\| \\
& =\sum_{k=1}^{\infty} \frac{K^{k-1}}{(k-1) !}\|W-Z\|=\sum_{k=0}^{\infty} \frac{K^{k}}{k !}\|W-Z\|=\mathrm{e}^{K}\|W-Z\|
\end{aligned}
$$

which was to prove.

Assumptions 9. Let $Q: \mathbb{R}^{d} \rightarrow \mathbb{R}^{d \times d}$ be a function which satisfies the following.

(a) The matrix $Q(w) \in \mathbb{R}^{d \times d}$ has nonpositive real eigenvalues.

(b) Function $Q$ is Lipschitz continuous for positive vectors, that is, there exists a constant $L_{Q} \geq 0$ such that $\| Q(v)-$ $Q(w)\left\|\leq L_{Q}\right\| v-w \|$ for all positive vectors $v, w \in \mathbb{R}^{d}$.

(c) Function $Q$ is bounded for a bounded set of positive vectors, that is, there exists a constant $M_{Q} \geq 0$ such that $\|Q(w)\| \leq M_{Q}$ holds for a bounded set of positive vectors $w \in \mathbb{R}^{d}$.

(d) Function $Q$ is twice differentiable with bounded derivatives for a bounded set of positive vectors. 
Proposition 10. Under Assumptions 9, the Magnus-type integrator (13) is convergent of second-order, that is, there exists a constant $C>0$, independent of $\tau$ and $n$, such that the error estimate $\left\|Y\left(t_{n}\right)-y_{n}\right\| \leq C \tau^{2}$ holds for all $n \in \mathbb{N}_{0}$ and $\tau \geq 0$ with $t_{n}=n \tau \in[0, T]$.

Proof. We first observe that the initial error vanishes. For all $n \in \mathbb{N}_{0}$, let $\widehat{y}_{n}$ be the solution defined in (8) with the operator $A$ defined in (10):

$$
\widehat{y}_{n+1}=\mathrm{e}^{\tau A\left(t_{n}+\frac{\tau}{2}\right)} \widehat{y}_{n}=\mathrm{e}^{\tau Q\left(Y\left(t_{n}-\delta+\frac{\tau}{2}\right)\right)} \widehat{y}_{n} .
$$

Since Assumptions 9 imply that the operator $A(t)=Q(Y(t-\delta))$ satisfies the assumptions of Theorem 3 with $a=0$, we have

$$
\left\|Y\left(t_{n+1}\right)-\widehat{y}_{n+1}\right\|=\mathcal{O}\left(\tau^{2}\right) .
$$

The triangular inequality yields the following estimate on the global error:

$$
\varepsilon_{n+1}:=\left\|Y\left(t_{n+1}\right)-y_{n+1}\right\| \leq\left\|Y\left(t_{n+1}\right)-\widehat{y}_{n+1}\right\|+\left\|\widehat{y}_{n+1}-y_{n+1}\right\|,
$$

where the first term on the right-hand side is $\mathcal{O}\left(\tau^{2}\right)$ by relation (16). Our aim is to estimate the second term on the right-hand side of (17). By using the telescopic identity we obtain the estimate

$$
\begin{aligned}
& \left\|\widehat{y}_{n+1}-y_{n+1}\right\|=\left\|\prod_{k=0}^{n} \mathrm{e}^{\tau Q\left(Y\left(t_{k}-\delta+\frac{\tau}{2}\right)\right)} Y_{0}-\prod_{k=0}^{n} \mathrm{e}^{\tau Q\left(\widetilde{y}_{k}\right)} Y_{0}\right\| \\
& \leq \sum_{j=0}^{n} \prod_{k=j+1}^{n}\left\|\mathrm{e}^{\tau Q\left(Y\left(t_{k}-\delta+\frac{\tau}{2}\right)\right)}\right\|\left\|\mathrm{e}^{\tau Q\left(Y\left(t_{j}-\delta+\frac{\tau}{2}\right)\right)}-\mathrm{e}^{\tau Q\left(\widetilde{y}_{j}\right)}\right\| \prod_{k=0}^{j-1}\left\|\mathrm{e}^{\tau Q\left(\widetilde{y}_{k}\right)}\right\|\left\|Y_{0}\right\|,
\end{aligned}
$$

where $\widetilde{y}_{k}$ was introduced in (13). By the assumption on the eigenvalues of $Q(w)$, we have $\left\|\mathrm{e}^{t Q(w)}\right\| \leq 1$ for all $t \geq 0$ and positive vectors $w \in \mathbb{R}^{d}$. Thus, we can further write that

$$
\left\|\widehat{y}_{n+1}-y_{n+1}\right\| \leq \sum_{j=0}^{n} \underbrace{\left\|\mathrm{e}^{\tau Q\left(Y\left(t_{j}-\delta+\frac{\tau}{2}\right)\right)}-\mathrm{e}^{\tau Q\left(\widetilde{y}_{j}\right)}\right\|}_{(*)}\left\|Y_{0}\right\|,
$$

where the term $(*)$ is a kind of local error to be estimated next. In what follows we use the exponential form of the matrices and the telescopic identity:

$$
\begin{aligned}
(*) & =\left\|\mathrm{e}^{\tau Q\left(Y\left(t_{j}-\delta+\frac{\tau}{2}\right)\right)}-\mathrm{e}^{\tau Q\left(\widetilde{y}_{j}\right)}\right\|=\sum_{m=0}^{\infty} \frac{1}{m !}\left\|\left(\tau Q\left(Y\left(t_{j}-\delta+\frac{\tau}{2}\right)\right)\right)^{m}-\left(\tau Q\left(\widetilde{y}_{j}\right)\right)^{m}\right\| \\
& \leq \sum_{m=0}^{\infty} \frac{1}{m !} \sum_{k=0}^{m-1}\left\|\tau Q\left(Y\left(t_{k}-\delta+\frac{\tau}{2}\right)\right)\right\|^{m-1-k}\left\|\tau Q\left(Y\left(t_{j}-\delta+\frac{\tau}{2}\right)\right)-\tau Q\left(\widetilde{y}_{j}\right)\right\|\left\|\tau Q\left(\widetilde{y}_{j}\right)\right\|^{k} \\
& \leq \sum_{m=0}^{\infty} \frac{1}{m !} \sum_{k=0}^{m-1} \tau^{m-1-k} M_{Q}^{m-1-k} \tau L_{Q} \underbrace{\left\|Y\left(t_{j}-\delta+\frac{\tau}{2}\right)-\widetilde{y}_{j}\right\|}_{\Delta_{j}} \tau^{k} M_{Q}^{k}=\sum_{m=0}^{\infty} \frac{\tau^{m} M_{Q}^{m-1}}{(m-1) !} L_{Q} \Delta_{j}=\tau \mathrm{e}^{\tau M_{Q}} L_{Q} \Delta_{j} .
\end{aligned}
$$

Here we used Assumptions $9 /(\mathrm{c})$ for the bounded sets $\{Y(t), t \in[0, T]\}$ and $\left\{\widetilde{y}_{n}, n \in \mathbb{N}_{0}\right.$ with $\left.n \tau \in[0, T]\right\}$ of positive vectors. Since $\mathrm{e}^{\tau M_{Q}}=\mathcal{O}(1)$, we have

$$
(*)=\mathcal{O}(\tau) \Delta_{j} .
$$

The term $\Delta_{j}$ can be computed from formulae (13) as

$$
\Delta_{j}= \begin{cases}0 & \text { for } j=0, \ldots, N-1 \\ \left\|Y\left(t_{j}-\delta+\frac{\tau}{2}\right)-\mathrm{e}^{\frac{\tau}{2} Q\left(\phi\left(t_{j}-2 \delta\right)\right)} y_{j-N}\right\| & \text { for } j=N, \ldots, 2 N-1 \\ \left\|Y\left(t_{j}-\delta+\frac{\tau}{2}\right)-\mathrm{e}^{\frac{\tau}{2} Q\left(y_{j-N}\right)} y_{j-N}\right\| & \text { for } j \geq 2 N .\end{cases}
$$

Since the exact solution $Y$ is given by an evolution family, we reformulate it as

$$
Y\left(t_{j}-\delta+\frac{\tau}{2}\right)=\mathrm{e}^{\Omega\left(t_{j}-\delta+\frac{\tau}{2}, 0\right)} Y_{0}=\mathrm{e}^{\Omega\left(t_{j}-\delta+\frac{\tau}{2}, t_{j}-\delta\right)} \mathrm{e}^{\Omega\left(t_{j}-\delta, 0\right)} Y_{0}=\mathrm{e}^{\Omega\left(t_{j}-\delta+\frac{\tau}{2}, t_{j}-\delta\right)} Y\left(t_{j}-\delta\right) .
$$

For $j=N, \ldots, 2 N-1$, we use the triangular inequality to get the estimate

$$
\Delta_{j} \leq \underbrace{\left\|\mathrm{e}^{\Omega\left(t_{j}-\delta+\frac{\tau}{2}, t_{j}-\delta\right)}-\mathrm{e}^{\frac{\tau}{2} Q\left(\phi\left(t_{j}-2 \delta\right)\right)}\right\|}_{(* *)} \underbrace{\left\|Y\left(t_{j}-\delta\right)\right\|}_{\leq M_{Y}}+\underbrace{\left\|\mathrm{e}^{\frac{\tau}{2} Q\left(\phi\left(t_{j}-2 \delta\right)\right)}\right\|}_{\leq 1} \underbrace{\left\|Y\left(t_{j}-\delta\right)-y_{j-N}\right\|}_{\varepsilon_{j-N}} .
$$


Here $Y_{M}$ is the bound on the exact solution over the compact time interval $[0, T]$. It exists because $Y$ is given by an evolution family which is strongly continuous, that is, $Y$ is a continuous function over $[0, T]$, hence, bounded. To bound the term $(* *)$, we use Lemma 8 and consider

$$
\begin{aligned}
& \left\|\Omega\left(t_{j}-\delta+\frac{\tau}{2}, t_{j}-\delta\right)-\frac{\tau}{2} Q\left(\phi\left(t_{j}-2 \delta\right)\right)\right\| \\
\leq & \left\|\Omega\left(t_{j}-\delta+\frac{\tau}{2}, t_{j}-\delta\right)-\int_{0}^{\tau / 2} Q\left(\phi\left(t_{j}-2 \delta+s\right)\right) \mathrm{d} s\right\|+\left\|\int_{0}^{\tau / 2} Q\left(\phi\left(t_{j}-2 \delta+s\right)\right) \mathrm{d} s-\frac{\tau}{2} Q\left(\phi\left(t_{j}-2 \delta\right)\right)\right\| .
\end{aligned}
$$

The definition (10) of operator $A$ and formula (5) with the choice $T=t_{n}-\delta, \Delta T=\frac{\tau}{2}$ leads to

$$
\int_{0}^{\tau / 2} Q\left(\phi\left(t_{j}-2 \delta+s\right)\right) \mathrm{d} s=\int_{0}^{\tau / 2} A\left(t_{j}-\delta+s\right) \mathrm{d} s=\Omega^{[1]}\left(t_{j}-\delta+\frac{\tau}{2}, t_{j}-\delta\right) .
$$

Hence, the first term on the right-hand side equals $\mathcal{O}\left(\tau^{2}\right)$ by Theorem 2 . Since the second term corresponds to the local error of the left rectangle rule, it is of $\mathcal{O}\left(\tau^{2}\right)$, too. Altogether we have

$$
\Delta_{j} \leq \mathcal{O}\left(\tau^{2}\right)+\varepsilon_{j-N} \text { for all } j=N, \ldots, 2 N-1 .
$$

For $j \geq 2 N$, we proceed similarly:

$$
\Delta_{j} \leq \underbrace{\left\|\mathrm{e}^{\Omega\left(t_{j}-\delta+\frac{\tau}{2}, t_{j}-\delta\right)}-\mathrm{e}^{\frac{\tau}{2} Q\left(y_{j-2 N}\right)}\right\|}_{(* * *)} \underbrace{\left\|Y\left(t_{j}-\delta\right)\right\|}_{\leq M_{Y}}+\underbrace{\left\|\mathrm{e}^{\frac{\tau}{2} Q\left(y_{j-2 N}\right)}\right\|}_{\leq 1} \underbrace{\left\|Y\left(t_{j}-\delta\right)-y_{j-N}\right\|}_{\varepsilon_{j-N}} .
$$

The term $(* * *)$ is approximated based on Lemma 8 as

$$
\begin{gathered}
\left\|\Omega\left(t_{j}-\delta+\frac{\tau}{2}, t_{j}-\delta\right)-\frac{\tau}{2} Q\left(y_{j-2 N}\right)\right\| \leq\left\|\Omega\left(t_{j}-\delta+\frac{\tau}{2}, t_{j}-\delta\right)-\int_{0}^{\tau / 2} Q\left(Y\left(t_{j}-2 \delta+s\right)\right) \mathrm{d} s\right\| \\
\quad+\left\|\int_{0}^{\tau / 2} Q\left(Y\left(t_{j}-2 \delta+s\right)\right) \mathrm{d} s-\frac{\tau}{2} Q\left(Y\left(t_{j}-2 \delta\right)\right)\right\|+\left\|\frac{\tau}{2} Q\left(Y\left(t_{j}-2 \delta\right)\right)-\frac{\tau}{2} Q\left(y_{j-2 N}\right)\right\| .
\end{gathered}
$$

As before, the first term is of $\mathcal{O}\left(\tau^{2}\right)$ by Theorem 2, the second is the local error of the left rectangle rule being $\mathcal{O}\left(\tau^{2}\right)$ as well, and the third term is $\mathcal{O}(\tau) \varepsilon_{j-2 N}$ by the Lipschitz continuity of $Q$. Altogether we have

$$
\Delta_{j} \leq \mathcal{O}\left(\tau^{2}\right)+\varepsilon_{j-N}+\mathcal{O}(\tau) \varepsilon_{j-2 N} \text { for all } j \geq 2 N .
$$

Substitution of estimates (20) and (21) into formulae (19), (18), and (17) yields

$$
\varepsilon_{n+1} \leq \mathcal{O}\left(\tau^{2}\right)+\mathcal{O}(\tau) \sum_{j=N}^{n} \varepsilon_{j-N}+\mathcal{O}\left(\tau^{2}\right) \sum_{j=2 N}^{n} \varepsilon_{j-2 N}
$$

for all $n \in \mathbb{N}_{0}$. Hence, we have the following cases.

For $n=0, \ldots, N-1$, we obtain $\varepsilon_{n+1}=\mathcal{O}\left(\tau^{2}\right)$.

For $n=N, \ldots, 2 N-1$, we have

$$
\varepsilon_{n+1}=\mathcal{O}\left(\tau^{2}\right)+\mathcal{O}(\tau) \sum_{j=N}^{n} \varepsilon_{j-N}
$$

where we use the previous case to obtain

$$
\varepsilon_{n+1}=\mathcal{O}\left(\tau^{2}\right)+\mathcal{O}(\tau) \underbrace{(n-N+1) \tau}_{t_{n-N+1}} \mathcal{O}(\tau)=\mathcal{O}\left(\tau^{2}\right) .
$$

And finally for $n \geq 2 N$, we use again the previous cases to obtain

$$
\varepsilon_{n+1}=\mathcal{O}\left(\tau^{2}\right)+\mathcal{O}(\tau) t_{n-N+1} \mathcal{O}(\tau)+\mathcal{O}\left(\tau^{2}\right) t_{n-2 N+1} \mathcal{O}(\tau)=\mathcal{O}\left(\tau^{2}\right) .
$$

Altogether we have $\varepsilon_{n+1}=\mathcal{O}\left(\tau^{2}\right)$ for all $n \in \mathbb{N}_{0}$ which was to show.

In what follows we apply the Magnus-type integrator (13) to delayed epidemic models.

\section{Magnus-type integrator for delayed epidemic models}

Let $S, I, R: \mathbb{R}_{0}^{+} \rightarrow[0,1]$ denote the proportion of susceptible, infected, and recovered humans among the total population, respectively. Their temporal change depends on various phenomena, from which we only consider now the infection-related ones. Then the number of susceptible individuals decreases because they are in contact with infected people and get infected. More precisely, the actual change in $S(t)$ depends on $S(t)$ itself and on that with how many 
infected people they met the latent period ago, that is, at time $t-\delta$. The number of infected individuals naturally increases with the same amount, and decreases with the number of people who recover. Based on this consideration a compartment-type model can be formulated.

Let $\beta>0$ denote the infection rate, $\gamma>0$ the recovery rate, and $\delta>0$ the latent period. Then for all $t>0$, we consider the simplest but most used delayed epidemic model (based on Kermack-McKendrick [2] and Cooke [13], but see also in [14-17], and the references therein):

$$
\left\{\begin{array}{l}
S^{\prime}(t)=-\beta S(t) \frac{I(t-\delta)}{1+\alpha I(t-\delta)}, \\
I^{\prime}(t)=\beta S(t) \frac{I(t-\delta)}{1+\alpha I(t-\delta)}-\gamma I(t), \\
R^{\prime}(t)=\gamma I(t),
\end{array}\right.
$$

where the value of the parameter $\alpha$ depends on what kind of model we deal with:

$$
\alpha= \begin{cases}0 & \text { for bilinear incidence rate, } \\ 1 & \text { for saturated incidence rate. }\end{cases}
$$

The incidence rate means the number of individuals who become infected in a unit of time. Initially, epidemic models were formulated by using the bilinear incidence rate (as in Kermack-McKendric model [2]). The saturated incidence rate was introduced in [18] (see also in [19]) but without taking into account the latent period. It contains the crowding effect of infective individuals, and tends to a saturation value when the proportion of infected individuals gets large.

The initial condition to problem (22) reads as

$$
S(0)=S_{0}, \quad I(0)=I_{0}, \quad R(0)=R_{0}, \quad \text { and } \quad I(s)=\varphi(s) \quad \text { for } s \in[-\delta, 0]
$$

with $S_{0}, I_{0}, R_{0} \geq 0$ given numbers and $\varphi:[-\delta, 0] \rightarrow \mathbb{R}_{0}^{+}$given continuous function. We also assume that $\varphi(0)=I_{0}$.

Let $Y: \mathbb{R}_{0}^{+} \rightarrow\left(\mathbb{R}_{0}^{+}\right)^{3}$ be defined as $Y(t)=(S(t), I(t), R(t))$ for all $t \geq 0$. Then the epidemic model (22) can be written as a quasilinear delay equation (9) with

$$
Q(w)=\left(\begin{array}{ccc}
-q\left(w^{(2)}\right) & 0 & 0 \\
q\left(w^{(2)}\right) & -\gamma & 0 \\
0 & \gamma & 0
\end{array}\right) \in \mathbb{R}^{3 \times 3} \quad \text { and } \quad q\left(w^{(2)}\right)=\frac{\beta w^{(2)}}{1+\alpha w^{(2)}}
$$

for any vector $w=\left(w^{(1)}, w^{(2)}, w^{(3)}\right) \in \mathbb{R}^{3}$. Since the matrix function $Q$ does not depend on the past values of $S$ and $R$, we can take $S(s)=S_{0}$ and $R(s)=R_{0}$ for $s \in[-\delta, 0)$ without the loss of generality. The initial function then reads as $\phi(s)=\left(S_{0}, \varphi(s), R_{0}\right)$ for $s \in[-\delta, 0]$.

Our aim is to apply the Magnus-type integrator (13) with $Q$ defined in (24). Therefore, we split the interval $[-\delta, 0]$ into $N \in \mathbb{N}$ pieces, and define the time step as $\tau=\delta / N$ such that it satisfies condition (12). Since $Q$ does only depend on the past values of $I$, we could take any values of $S_{n-N}$ and $R_{n-N}$ for $n<N$. In order to agree with the initial function $\phi$, we consider $S_{n-N}=S_{0} \geq 0$ and $R_{n-N}=R_{0} \geq 0$.

In what follows we prove the positivity preservation and the convergence of the Magnus-type integrator (13) with $Q$ defined in (24).

Since epidemic models deal with population, it is natural to assume that the values of $S(t), I(t), R(t)$ are nonnegative for all time $t \geq 0$. Thus, we expect the same from the numerical method as well, that is, $S_{n}, I_{n}, R_{n} \geq 0$ should hold for all $n \in \mathbb{N}_{0}$.

Proposition 11. The Magnus-type integrator (13) applied to delayed epidemic model (22) preserves the positivity.

Proof. By Proposition 7 it suffices to show that $Q(w) \in \mathbb{R}^{3 \times 3}$ is a Metzler matrix for all positive vectors $w \in \mathbb{R}^{3}$. Since $\alpha, \beta, \gamma>0$ holds and $w$ is a positive vector, the off-diagonal elements of $Q(w)$ in (24) are nonnegative.

To prove the convergence we will need the following properties of the function $Q$.

Proposition 12. Let $Q: \mathbb{R}^{d} \rightarrow \mathbb{R}^{d \times d}$ be the matrix function defined in (24).

(a) The eigenvalues of matrix $Q(w) \in \mathbb{R}^{3 \times 3}$ are nonpositive real numbers.

(b) The matrix function $Q$ is Lipschitz continuous for positive vectors.

(c) The estimate $\|Q(w)\| \leq \beta w^{(2)}+\gamma$ holds for all positive vectors $w=\left(w^{(1)}, w^{(2)}, w^{(3)}\right) \in \mathbb{R}^{3}$.

(d) For a bounded set $\left\{w_{n} \in\left(\mathbb{R}_{0}^{+}\right)^{3}, n \in \mathbb{N}\right\}$ of positive vectors, we have $\left\|Q\left(w_{n}\right)\right\| \leq M_{Q}$ for all $n \in \mathbb{N}_{0}$ with some bound $M_{Q} \geq 0$.

(e) The matrix function $Q$ is twice differentiable and estimates $\left\|Q^{\prime}(w)\right\| \leq \beta,\left\|Q^{\prime \prime}(w)\right\| \leq 2 \alpha \beta$ hold for all positive vectors $w \in \mathbb{R}^{3}$. 
Proof.

(a) Since matrix $Q(w)$, defined in (24), is a lower triangular matrix, its eigenvalues are the following:

$$
\lambda_{1}=0, \quad \lambda_{2}=-\gamma, \quad \lambda_{3}=-\frac{\beta w^{(2)}}{1+\alpha w^{(2)}} .
$$

The positivity of vector $w$ implies that $w^{(2)} \geq 0$, that is, all the eigenvalues above are nonpositive real numbers.

(b) Let $w=\left(w^{(1)}, w^{(2)}, w^{(3)}\right) \in \mathbb{R}^{3}$ and $z=\left(z^{(1)}, z^{(2)}, z^{(3)}\right) \in \mathbb{R}^{3}$ be arbitrary positive vectors. With functions $Q$ and $q$ defined in (24) we have

$$
\begin{aligned}
\|Q(w)-Q(z)\| & =\left\|\left(\begin{array}{ccc}
-\left(q\left(w^{(2)}\right)-q\left(z^{(2)}\right)\right) & 0 & 0 \\
q\left(y^{(2)}\right)-q\left(w^{(2)}\right) & 0 & 0 \\
0 & 0 & 0
\end{array}\right)\right\|=\left|q\left(w^{(2)}\right)-q\left(z^{(2)}\right)\right| \\
& =\left|\beta\left(\frac{w^{(2)}}{1+\alpha w^{(2)}}-\frac{z^{(2)}}{1+\alpha z^{(2)}}\right)\right|=\beta\left|\frac{w^{(2)}-z^{(2)}}{\left(1+\alpha w^{(2)}\right)\left(1+\alpha z^{(2)}\right)}\right| .
\end{aligned}
$$

Since $\alpha \in\{0,1\}$ and $w^{(2)}, z^{(2)}$ are nonnegative real numbers, the denominator is greater than or equal to one. Thus we have the estimate

$$
\|Q(w)-Q(z)\| \leq \beta \cdot\left|w^{(2)}-z^{(2)}\right| \leq \beta \cdot \max _{i=1,2,3}\left|w^{(i)}-z^{(i)}\right|=\beta \cdot\|w-z\|
$$

proving the Lipschitz continuity of $Q$ for positive vectors.

(c) For a positive vector $w \in \mathbb{R}^{3}$ we have

$$
\begin{aligned}
\|Q(w)\| & =\left\|\left(\begin{array}{ccc}
-q\left(w^{(2)}\right) & 0 & 0 \\
q\left(w^{(2)}\right) & -\gamma & 0 \\
0 & -\gamma & 0
\end{array}\right)\right\|=q\left(w^{(2)}\right)+\gamma \\
& =\frac{\beta w^{(2)}}{1+\alpha w^{(2)}}+\gamma \leq \beta w^{(2)}+\gamma
\end{aligned}
$$

which was to be shown.

(d) The previous statement (c) implies that for a bounded set $\left\{w_{n} \in\left(\mathbb{R}_{0}^{+}\right)^{3}, n \in \mathbb{N}\right\}$ of positive vectors we have $\left\|Q\left(w_{n}\right)\right\| \leq M_{Q}$ for all $n \in \mathbb{N}_{0}$ with some bound $M_{Q} \geq 0$.

(e) Let $w=\left(w^{(1)}, w^{(2)}, w^{(3)}\right) \in \mathbb{R}^{3}$ be an arbitrary positive vector. Since $Q$ maps from $\mathbb{R}^{3}$ into $\mathbb{R}^{3 \times 3}$, its derivative $Q^{\prime}$ can be represented as a continuous and linear mapping from $\mathbb{R}^{3}$ to $\mathbb{R}^{9}$, which has only two non-zero elements $\pm q^{\prime}\left(w^{(2)}\right)$ and exists for all positive $w$ vectors. The local boundedness follows from the nonnegativity of $\alpha$ and $w^{(2)}$ :

$$
\left\|Q^{\prime}(w)\right\|=\left|q^{\prime}\left(w^{(2)}\right)\right|=\frac{\beta}{\left|\left(1+\alpha w^{(2)}\right)^{2}\right|} \leq \beta .
$$

Similarly, for the second derivative we have the bound

$$
\left\|Q^{\prime \prime}(w)\right\|=\left|q^{\prime \prime}\left(w^{(2)}\right)\right|=\frac{2 \alpha \beta}{\left|\left(1+\alpha w^{(2)}\right)^{3}\right|} \leq 2 \alpha \beta .
$$

These bounds were to be proved.

We show now the convergence of the Magnus-type integrator (13) when applied to the delayed epidemic model (22). We only need to check whether the assumptions in Proposition 10 are fulfilled for the function $Q$ defined by formula (24).

Proposition 13. The Magnus-type integrator (13) is convergent of second-order when applied to the delayed epidemic model (22).

Proof. We have to check Assumptions 9 for the matrix function $Q: \mathbb{R}^{3} \rightarrow \mathbb{R}^{3 \times 3}$ defined in (24).

(a) Proposition 12/(a) states that the eigenvalues of $Q(w)$ are nonpositive real numbers for all positive vectors $w$.

(b) The Lipschitz continuity of $Q$ follows from Proposition $12 /(\mathrm{b})$.

(c) The boundedness of $Q(w)$ follows from Proposition $12 /(\mathrm{d})$.

(d) The differentiability and the boundedness of the derivatives follow from $12 /(\mathrm{e})$.

Proposition 10 implies now the convergence. 


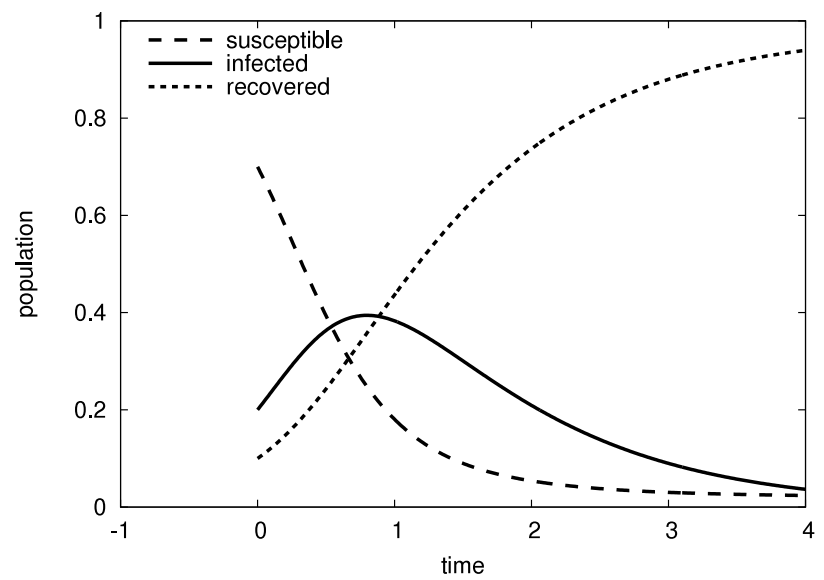

Fig. 1. Numerical solution of the epidemic model (22) without taking into account the latent period $(\delta=0)$ with parameters are $\alpha=0, \beta=4$, $\gamma=1$, initial values $S_{0}=0.7, I_{0}=0.2, R_{0}=0.1$, and time step $\tau=0.01$.

\section{Numerical experiments}

We illustrate our convergence result by numerical experiments done for the delayed epidemic model (22) with $\alpha=0$. Since in the computer code the initial function $\phi$ is given as a vector with entries $\phi\left(t_{n}-\delta\right), n=0, \ldots, N$, we use the approximation

$$
\phi\left(t_{n}-\delta+\frac{\tau}{2}\right) \approx \frac{1}{2}\left(\phi\left(t_{n}-\delta\right)+\phi\left(t_{n}-\delta+\tau\right)\right) \text { for } n=0, \ldots, N-1
$$

in formula (13). We note that this approximation does not have any influence on the order of the Magnus-type integrator.

\subsection{Role of latent period}

Fig. 1 shows the solution to epidemic model (22) without taking into account the latent period, i.e., $\delta=0$. We take the parameters $\alpha=0, \beta=4$, initial values $S_{0}=0.7, I_{0}=0.2, R_{0}=0.1$, and time step $\tau=0.01$.

In Figs. 2 and 3 we present two examples of the solution to the delayed epidemic model (22) with the same parameters, initial values, and time step as before. The latent period $\delta=1$ is incorporated in the equations with the respective history functions

$$
\begin{aligned}
& \varphi(s)=I_{0}, \\
& \varphi(s)=I_{0}+\frac{1}{2} s
\end{aligned}
$$

for all $s \in[-\delta, 0]$ in (23). One can see that already the constant history function changes the temporal behavior of the solution: the maximal number of infected individuals is less than in the case without latent period (cf. Fig. 2). The linearly increasing history function introduces a new phenomena: The proportion of infected humans decreases at the beginning of the time interval (cf. Fig. 3).

The importance of considering the latent period is made clear by Figs. 4 and 5 with $\beta=1$ and the history function

$$
\varphi(s)=I_{0}-\frac{1}{2} s, \quad s \in[-\delta, 0] .
$$

Fig. 4 shows the solution when the latent period is not taken into account (i.e. $\delta=0$ ). In this case the model does not forecast any epidemic: The proportion of infected individuals monotonically decreases. However, when the latent period is incorporated in the equations, the proportion of infected individuals starts to increase, that is, an epidemic occurs. The function $I$ decreases only after the proportion $S$ of susceptible humans reaches the critical value

$$
S_{\mathrm{c}}=\frac{\gamma}{\beta} \frac{1+\alpha I\left(t_{\mathrm{c}}-\delta\right)}{I\left(t_{\mathrm{c}}-\delta\right)} I\left(t_{\mathrm{c}}\right)
$$

where $t_{\mathrm{c}}$ denote the time when $S\left(t_{\mathrm{c}}\right)=S_{\mathrm{c}}$. The difference between the two cases is caused by the delay. When there is a latent period $\delta>0$ in Fig. 5, the actual derivative of $I$ depends on how many susceptible humans there are now and how many infected humans there were the latent period ago because latter individuals begin to infect at present. 


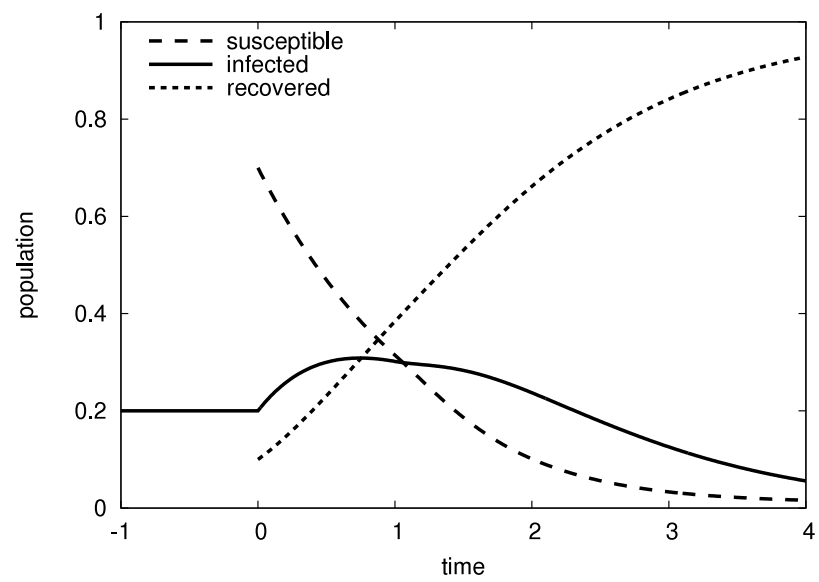

Fig. 2. Numerical solution of delayed epidemic model (22) with parameters $\alpha=0, \beta=4, \gamma=1$, initial values $S_{0}=0.7, I_{0}=0.2, R_{0}=0.1$, and time step $\tau=0.01$ with latent period $\delta=1$ and history functions (26).

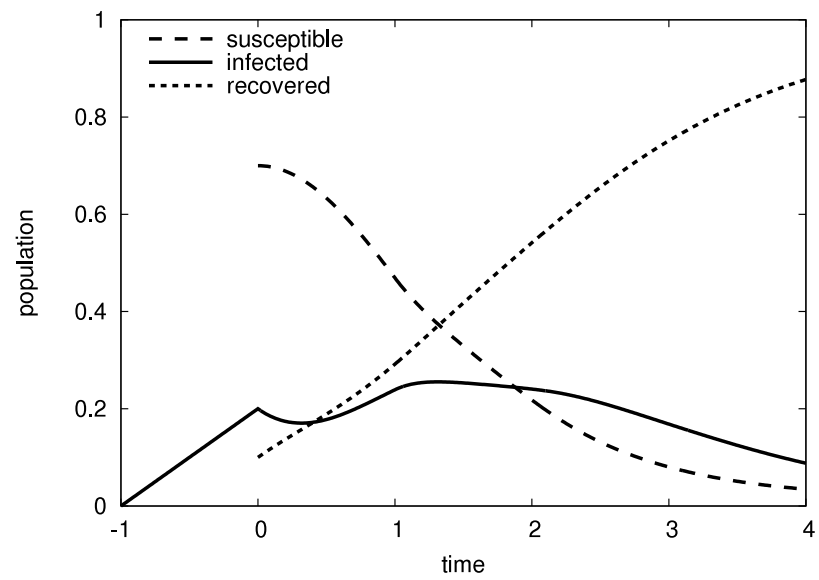

Fig. 3. Numerical solution of delayed epidemic model (22) with parameters $\alpha=0, \beta=4, \gamma=1$, initial values $S_{0}=0.7, I_{0}=0.2, R_{0}=0.1$, and time step $\tau=0.01$ with latent period $\delta=1$ and history functions (27).

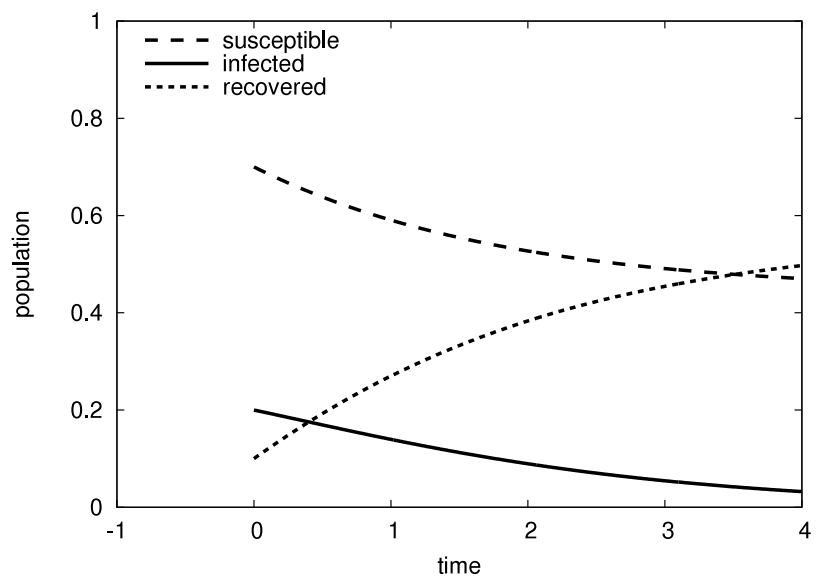

Fig. 4. Numerical solution of delayed epidemic model (22) with parameters $\alpha=0, \beta=1, \gamma=1$, history function (28), and time step $\tau=0.01$ without taking into account the latent period. 


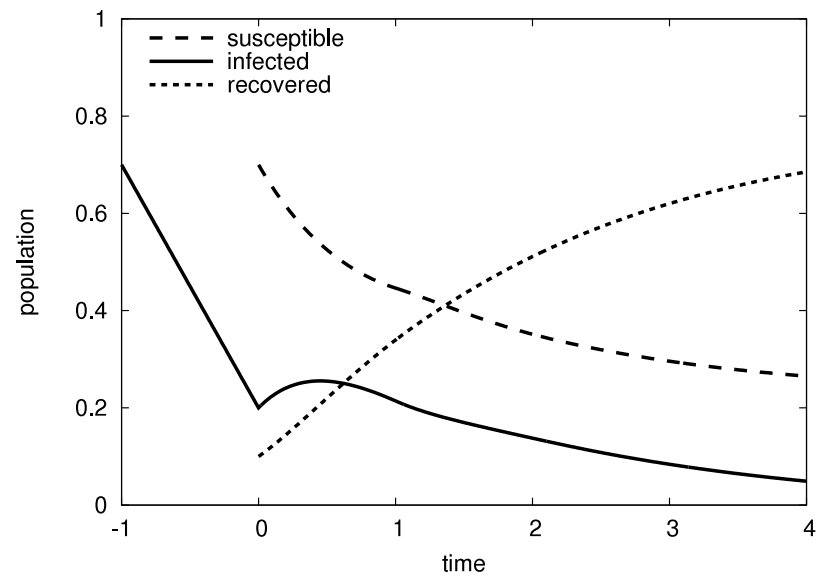

Fig. 5. Numerical solution of delayed epidemic model (22) with parameters $\alpha=0, \beta=1, \gamma=1$, history function (28), and time step $\tau=0.01$ with latent period $\delta=1$.

Table 1

Slopes $p$ of the fitted line which corresponds to the numerical order of the Magnus-type integrator. The numerical solution is compared to a reference solution with time step $\tau_{\text {ref }}$ computed by the Magnus-type integrator itself.

\begin{tabular}{llll}
\hline$\varphi(s)$ & $\tau_{\text {ref }}$ & $\# \tau$ & $p$ \\
\hline$(26)$ & $10^{-5}$ & 18 & 1.96688 \\
$(26)$ & $10^{-4}$ & 15 & 1.98539 \\
$(27)$ & $10^{-4}$ & 15 & 1.99205 \\
$(28)$ & $10^{-4}$ & 15 & 1.99653 \\
\hline
\end{tabular}

\subsection{Error analysis}

We perform the error analysis of the Magnus-type integrator (13) by comparing its solution to a reference solution $y_{\text {ref }}$ being accurate enough to be treated as a good approximation of the exact solution $y$. We computed the reference solution with the following three methods:

(a) the Magnus-type integrator (13) itself with small reference time step $\tau_{\text {ref, }}$,

(b) the built-in function dde23. $\mathrm{m}$ of the programming environment MATLAB which uses adaptive step size and is of order (2)3,

(c) the FORTRAN function retard.f written by E. Hairer which uses the Dormand-Prince variable step size method of order (4)5 and is available under the link [20] (for more details see [21]).

In all cases the relative global error

$$
\varepsilon(\tau)=\frac{\left\|y_{\text {ref }}\left(t_{\max }\right)-y_{n_{\max }}\right\|}{\left\|y_{\text {ref }}\left(t_{\max }\right)\right\|}
$$

is computed and plotted in logarithmic scale. Here $t_{\max }$ refers to the maximal time level and $n_{\max }$ to its index. Then the slope of the line fitted onto the numerical results corresponds to the approximate value of the order of convergence. The various values of the time step are specified as $\tau_{j}=\sqrt{5} \cdot \tau_{j-1}$ for $j=2, \ldots, \# \tau$ with $\tau_{1}=0.2$.

Fig. 6 shows the relative global error (29) and the fitted line when the reference solution is computed with the Magnustype integrator itself with time step $\tau=10^{-4}$. The slope $1.98539 \approx 2$ of the fitted line represents the numerical value of the convergence order proved in Proposition 13. Table 1 displays the slopes of the fitted lines for the various history functions. Since all of them are approximately 2 , the numerical results illustrate well the second-order convergence.

Next we present our results when the reference solution is computed by functions dde23.m and retard.f with the same parameters as before. Since both methods are using adaptive time steps, we can only control the values of the relative and the maximum tolerance. The corresponding order plots are shown in Fig. 7. The role of the tolerance is clearly seen: Once the accuracy of the Magnus-type integrator reaches the accuracy of the reference solution, the relative global error's formula (29) does not work anymore. Thus, the line should be fitted only to the points before this limit. Table 2 shows the corresponding slopes which are approximately 2 also in these cases. 


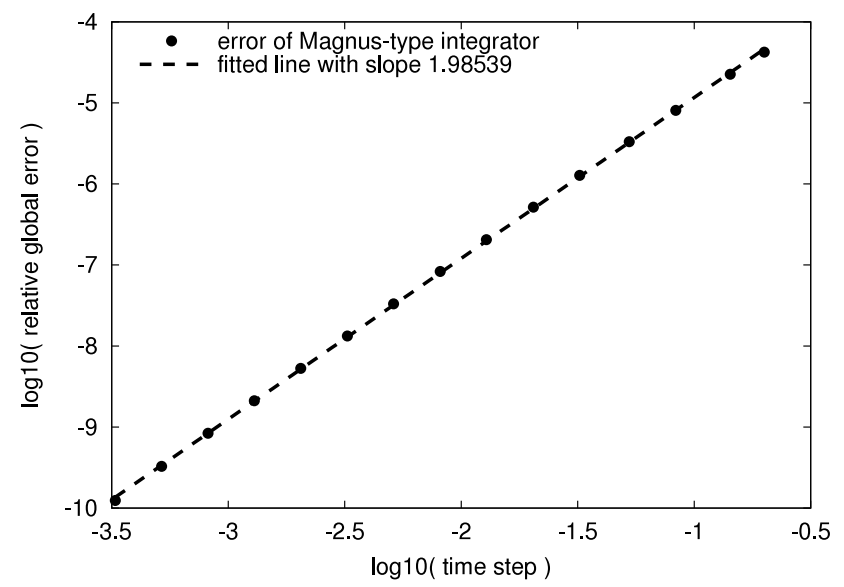

Fig. 6. Order plot of Magnus-type integrator (13) when applied to the delayed epidemic model (22) with $\alpha=0, \beta=4, \gamma=1, \delta=1, \tau_{\text {ref }}=10^{-4}$ and history function (27). The dots correspond to the relative global error (29) of the model. The slope $1.98539 \approx 2$ of the fitted line represents the numerical value of the convergence order.

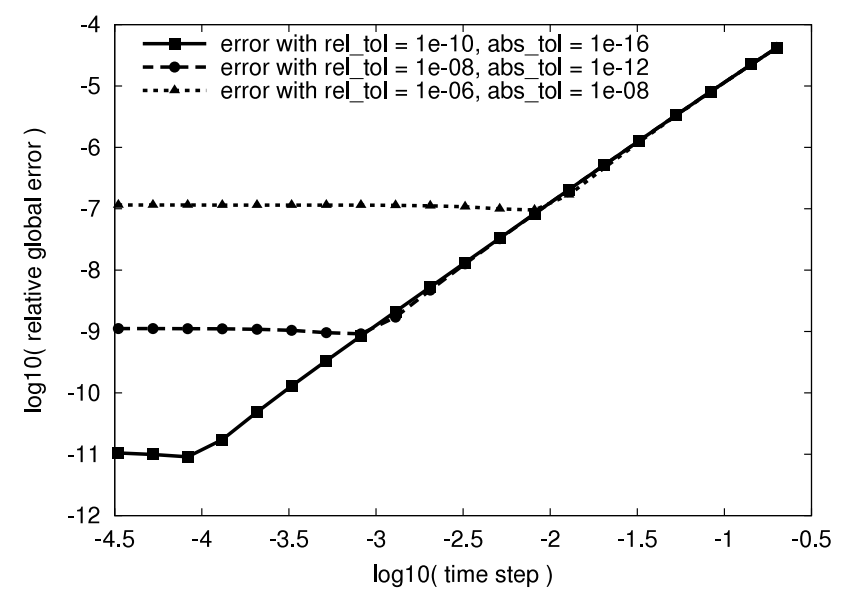

Fig. 7. Order plot of Magnus-type integrator (13) when applied to the delayed epidemic model (22) with $\alpha=0, \beta=4, \gamma=1, \delta=1$, history function (26). The reference solution is computed by function dde $23 . \mathrm{m}$ with various values of relative and maximum tolerance. The dots correspond to the relative global error (29) of the model.

Table 2

Slopes $p$ of the fitted line which corresponds to the numerical order of the Magnus-type integrator compared to functions dde23.m and retard.f with various relative and absolute tolerance values and numbers of time steps.

\begin{tabular}{lllrll}
\hline$\varphi(s)$ & rel_tol & abs_tol & $\# \tau$ & $p$ (dde23.m) & $p$ (retard.f) \\
\hline$(26)$ & $1 \mathrm{e}-10$ & $1 \mathrm{e}-16$ & 18 & 1.99455 & 1.96882 \\
$(26)$ & $1 \mathrm{e}-08$ & $1 \mathrm{e}-12$ & 13 & 1.98753 & 1.97601 \\
$(26)$ & $1 \mathrm{e}-06$ & $1 \mathrm{e}-08$ & 8 & 1.95709 & 1.94260 \\
$(27)$ & $1 \mathrm{e}-08$ & $1 \mathrm{e}-12$ & 13 & 1.97376 & 1.97948 \\
$(28)$ & $1 \mathrm{e}-08$ & $1 \mathrm{e}-12$ & 13 & 2.00972 & 1.98551 \\
\hline
\end{tabular}

\subsection{Summary}

Both the theoretical and the numerical results show that the Magnus-type integrator offers an efficient way of solving delayed epidemic models. Namely, it is a second-order method, so it gives a more accurate result than explicit or implicit Euler method which are usually used in population dynamics when one wishes to write an own code. Moreover, it is an explicit method, therefore, it does not need the solution of a system of nonlinear algebraic equations. We remark that the stability issue (which is usually solved by applying an implicit method) is not relevant here due to the exact computation of the matrix exponential. Therefore, the explicit kind of the method is not a disadvantage anymore, but on the contrary: it makes the computations fast and the implementation straightforward. 
In a forthcoming paper we aim at generalizing our results concerning the second-order convergence of Magnus-type integrator to the case when it is applied to abstract semilinear delay equations corresponding to partial differential equations. We will work in the framework of linear evolution families which is an efficient tool for treating nonautonomous equations on appropriate function spaces. We plan to study the higher order case as well.

\section{Acknowledgments}

The project was supported by the Hungarian National Research, Development and Innovation Office (NKFIH) under the grants PD-121117 and SNN-125119. The author acknowledges the Bolyai János Research Scholarship of the Hungarian Academy of Sciences.

\section{References}

[1] M. Martcheva, An Introduction to Mathematical Epidemiology, Springer, 2015.

[2] W. Kermack, A. McKendrick, A contribution to the mathematical theory of epidemics, Proc. R. Soc. A 115 (1927) $700-721$.

[3] W. Magnus, On the exponential solution of a differential equation for a linear operator, Comm. Pure Appl. Math. 7 (1954) 649-673.

[4] G. Nickel, Evolution semigroups for nonautonomous Cauchy problems, Abstr. Appl. Anal. 2 (1997) 73-95.

[5] A. Bátkai, P. Csomós, B. Farkas, Operator splitting for nonautonomous delay equations, Comput. Math. Appl. 65 (2013) $315-324$.

[6] A. Iserles, S.P. Nørsett, On the solution of linear differential equations in Lie groups, Philos. Trans. R. Soc. Lond. A Math. Phys. Eng. Sci. 357 (1999) 983-1019.

[7] S. Blanes, F. Casas, J.A. Oteo, J. Ros, Magnus and Fer expansions for matrix differential equations: the convergence problem, J. Phys. A: Math. Gen. 31 (1998) 259-268.

[8] S. Blanes, F. Casas, J.A. Oteo, J. Ros, The Magnus expansion and some of its applications, Phys. Rep. 470 (2009) $151-238$.

[9] P.C. Moan, J. Niesen, Convergence of the magnus series, J. Found. Comput. Math. 8 (2008) $291-301$.

[10] F. Casas, A. Iserles, Explicit Magnus expansions for nonlinear equations, J. Phys. A: Math. Gen. 39 (2006) $5445-5462$.

[11] C. González, A. Ostermann, M. Thalhammer, A second-order Magnus-type integrator for nonautonomous parabolic problems, J. Comput. Appl. Math. 189 (2006) 142-156.

[12] A. Bátkai, M. Kramar Fijavž, A. Rhandi, Positive Operator Semigroups, from Finite to Infinite Dimensions, Birkhäuser, 2017.

[13] K.L. Cooke, Stability analysis for a vector disease model, Rocky Mountain J. Math. 9 (1979) $31-42$.

[14] V. Capasso, G. Serio, A generalization of the Kermack-Mckendrick deterministic epidemic model, Math. Biosci. 42 (1978) $41-61$.

[15] W. Ma, M. Song, Y. Takeuchi, Global stability of an SIR epidemic model with time delay, Appl. Math. Lett. 17 (2004) $1141-1145$.

[16] C.C. McCluskey, Global stability for an SIR epidemic model with delay and nonlinear incidence, Nonlinear Anal. RWA 11 (2010) $3106-3109$.

[17] R. Xu, Z. Ma, Global stability of a SIR epidemic model with nonlinear incidence rate and time delay, Nonlinear Anal. RWA 10 (2009) 3175-3189.

[18] R.M. Anderson, R.M. May, Regulation and stability of host-parasite population interactions: I. regulatory processes, J. Anim. Ecol. 47 (1978) 219-267.

[19] L. Esteva, M. Matias, A model for vector transmitted diseases with saturation incidence, J. Biol. Systems 9 (2001) $235-245$.

[20] Link to the FORTRAN function written by E. Hairer, 1995. http://www.unige.ch/ hairer/prog/nonstiff/retard.f.f.

[21] E. Hairer, S.P. Nørsett, G. Wanner, Solving Ordinary Differential Equations I. Nonstiff Problems, Springer-Verlag, 2008. 